\title{
A Novel Design for a Palm Prints Enabled Biometric System
}

\author{
Sulabh Kumra. Tanmay Rao \\ Department of Electronics and Instrumentation Engineering, ITM University, Gurgaon, India
}

\begin{abstract}
In this paper we are providing an approach for authentication using palm prints. Biometrics is physiological characteristics of human beings, unique for every individual that are usually time invariant and easy to acquire. Palm print is one of the relatively new physiological biometrics due to its stable and unique characteristics. In this paper, a combination of hand geometry and palmprint verification system is being developed. This system uses a scanner as sole sensor to obtain the hands images. First, the hand geometry verification system performs the feature extraction to obtain the geometrical information of the fingers and palm. Second, the region of interest (ROI) is detected and cropped by palmprint verification system. This ROI acts as the base for palmprint feature extraction by using Linear Discriminant Analysis (LDA). Lastly, the matching scores of the two individual classifiers is fused by several fusion algorithms namely sum rule, weighted sum rule and Support Vector Machine (SVM). The results of the fusion algorithms are being compared with the outcomes of the individual palm and hand geometry classifiers. We are able to show that fusion using SVM with Radial Basis Function (RBF) kernel has outperformed other combined and individual classifiers.
\end{abstract}

Keywords: Palm prints, fusion, palmprint, hand geometry.

\section{Introduction}

It is basically a pattern-recognition system that is used to identify or verify users based on his on her unique physical characteristics. Biometric systems offer several advantages over traditional authentication methods. Biometric information cannot be acquired by direct covert observation. It is impossible to share and difficult to reproduce. It enhances user convenience by alleviating the need to memorize long and random passwords. It protects against repudiation by the user. Biometrics provides the same level of security to all users unlike passwords and is highly resistant to brute force attacks. Moreover, biometrics is one of the few techniques that can be used for negative recognition where the system determines whether the person is who he or she denies to be. Using biometrics with password protected smart cards introduces all three factors of authentication simultaneously (something you know, something you have and something you are).

\subsection{Basic structure of a biometric system}

Every biometric system consists of four basic modules:

\subsubsection{Enrollment Unit}

The enrollment module registers individuals into the biometric system database. During this phase, a biometric reader scans the individual's biometric characteristic to produce its digital representation.

\subsubsection{Feature Extraction Unit}

Module processes the input sample to generate a compact representation called the template, which is then stored in a central database or a smartcard issued to the individual.

\subsubsection{Matching Unit}

This module compares the current input with the template. If the system performs identity verification, it compares the new characteristics to the user's master template and produces a score or match value (one to one matching). A system performing identification matches the new characteristics against the master templates of many users resulting in multiple match values (one to many matching).

\subsubsection{Decision Maker}

This module accepts or rejects the user based on a security threshold and matching score. 


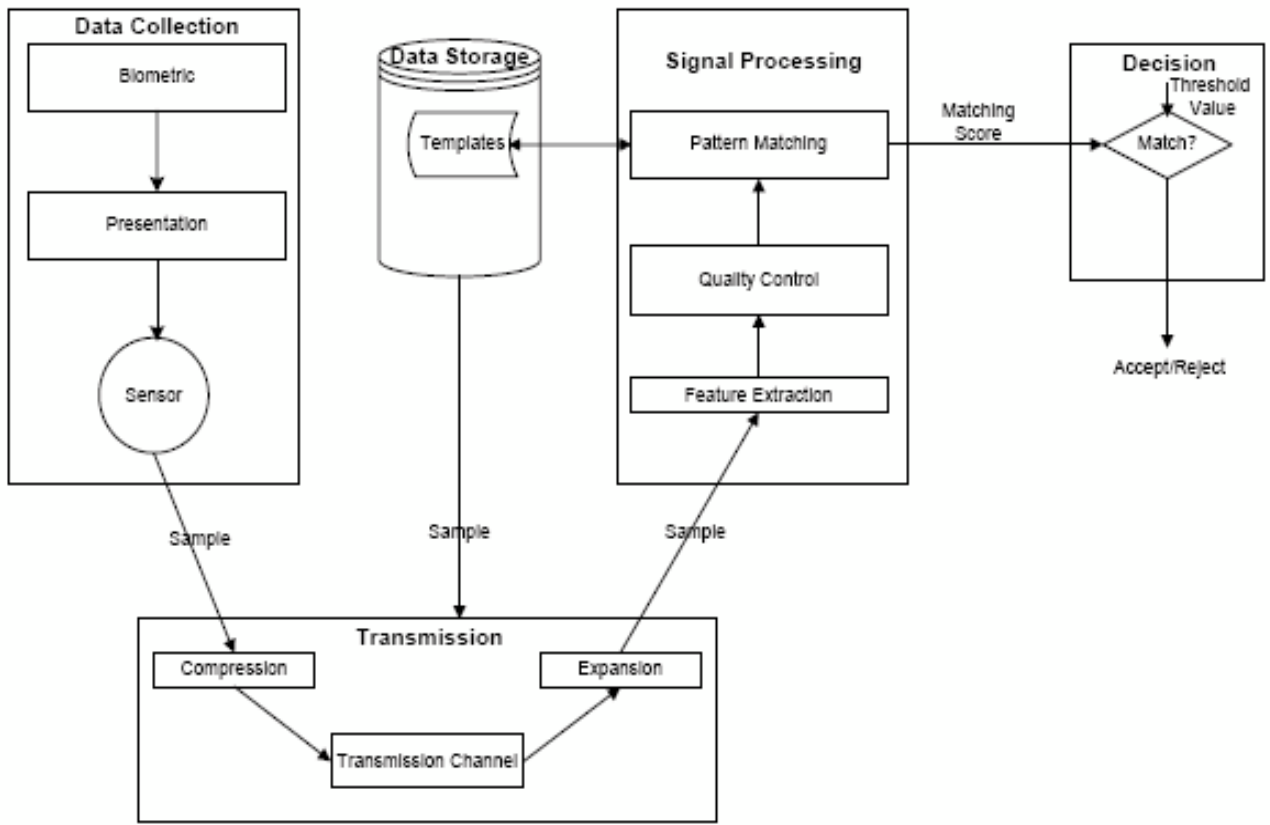

Fig. 1: Basic Structure of a Biometric Authentication System.

\section{Enrollment}

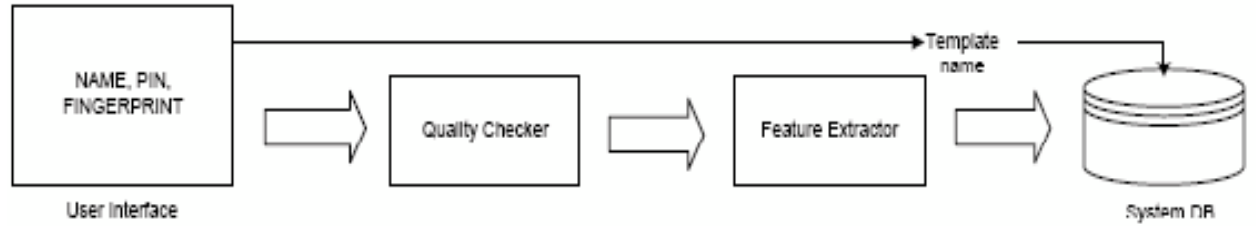

Verification

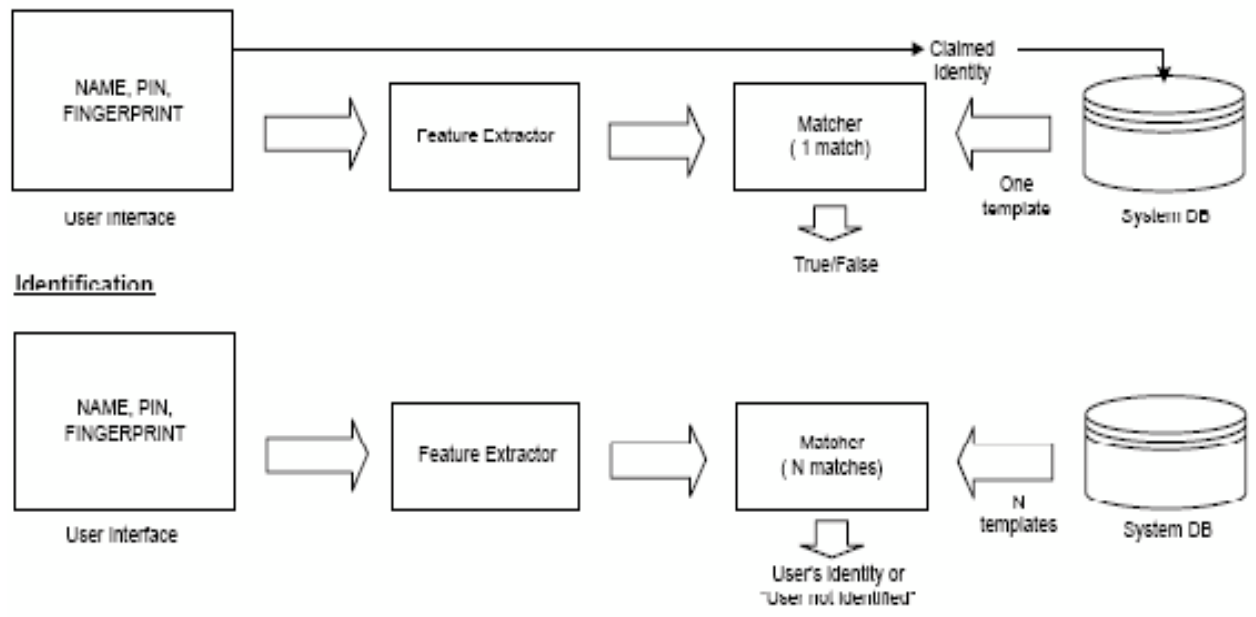

Fig. 2 : Enrollment, Identification and Verification in a Biometric System.

Biometric based personal identification is getting wide acceptance in the networked society, replacing passwords and keys due to its reliability, uniqueness and the ever increasing demand of security. Palm print is a new biometric modality which can be used for authentication of a person's identity because of its richness. Palm print not only has the information available on the fingerprint but it has far more amount of details in terms of principal lines, wrinkles and creases. Moreover it can easily be combined with hand shape biometric so as to form a highly accurate and reliable biometric based personal identification system. This type of identification has become an increasingly active research topic over the years. It has been analyzed for discriminating features like principal lines [1], geometry and texture [2]. 


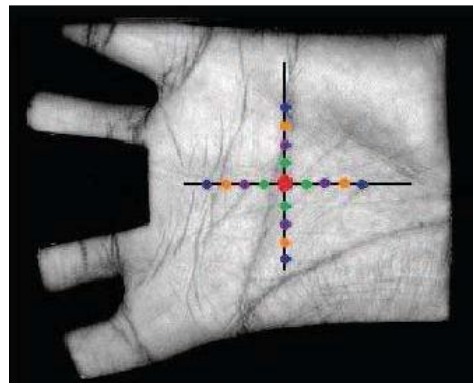

Fig. 3: Palm with Master and Slave points

The inner surface of the palm normally contains three flexion creases, secondary creases and ridges. The flexion creases are also called principal lines and the secondary creases are called wrinkles. The flexion and the major secondary creases are formed between the 3rd and 5th months of pregnancy [6] and superficial lines appear after we born. Although the three major flexions are genetically dependent, most of other creases are not [4]. Even identical twins have different palm prints [3]. These non-genetically deterministic and complex patterns are very useful in personal identification. Human beings were interested in palm lines for fortune telling long time ago. Scientists know that palm lines are associated with some genetic diseases including Down syndrome, Aarskog syndrome, Cohen syndrome and fetal alcohol syndrome [7]. Palm print research employs either high resolution or low resolution images. High resolution images are suitable for forensic applications such as criminal detection [5]. Low resolution images are more suitable for civil and commercial applications such as access control. Generally speaking, high resolution and a low resolution palmprint image. Researchers can extract ridges, singular points and minutia points as features from high resolution images while in low resolution images they generally extract principal lines, wrinkles and texture.refers to $400 \mathrm{dpi}$ or more and low resolution refers to $150 \mathrm{dpi}$ or less. Fig. 2 illustrates a part of a highresolution palmprint image Initially palmprint research focused on high-resolution images [8-10] but now almost all research is on low resolution images for civil and commercial applications. This is also the focus of this paper. The design of a biometric system takes account of five objectives: cost, user acceptance and environment constraints, accuracy, computation speed and security (Fig. 4). Reducing accuracy can increase speed. Typical examples are hierarchical approaches. Reducing user acceptance can improve accuracy. For instance, users are required to provide more samples for training. Increasing cost can enhance security. We can embed more sensors to collect different signals for liveness detection. In some applications, environmental constraints such as memory usage, power consumption, size of templates and size of devices have to be fulfilled. A biometric system installed in PDA (personal digital assistant) requires low power and memory consumption but these requirements may not be vital for biometric access control systems. A practical biometric system should balance all these aspects.

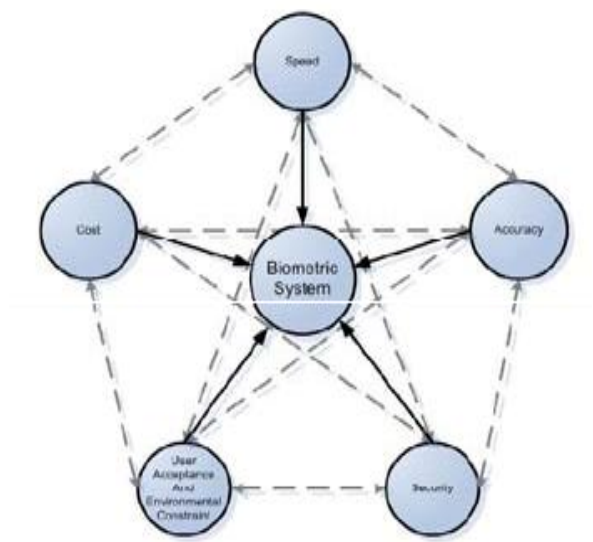

Fig. 4: Computation speed and security of Biometric system

Typical palm print recognition system consists of five parts: palm print scanner, preprocessing, feature extraction, matcher and database illustrated in Fig. 5. The palm print scanner collects palm print images. Preprocessing sets up a coordinate system to align palm print images and to segment a part of palm print image for feature extraction. Feature extraction obtains effective features from the preprocessed palm prints. A matcher compares two palm print features and a database stores registered templates. 


\section{Proposed Method}

The proposed method combines two biometric modalities, namely palmprint and hand geometry verification system. Only one hand image is captured during the image acquiring process. The palmprint and hand geometry features are simultaneously extracted using the same hand image. The Euclidean distance classifier is used to classify both individual hand geometry and palmprint features. Sum rule, weighted sum rule and SVM were used as decision level fusions to fuse the matching scores obtained from the hand geometry and palmprint individual classifiers.

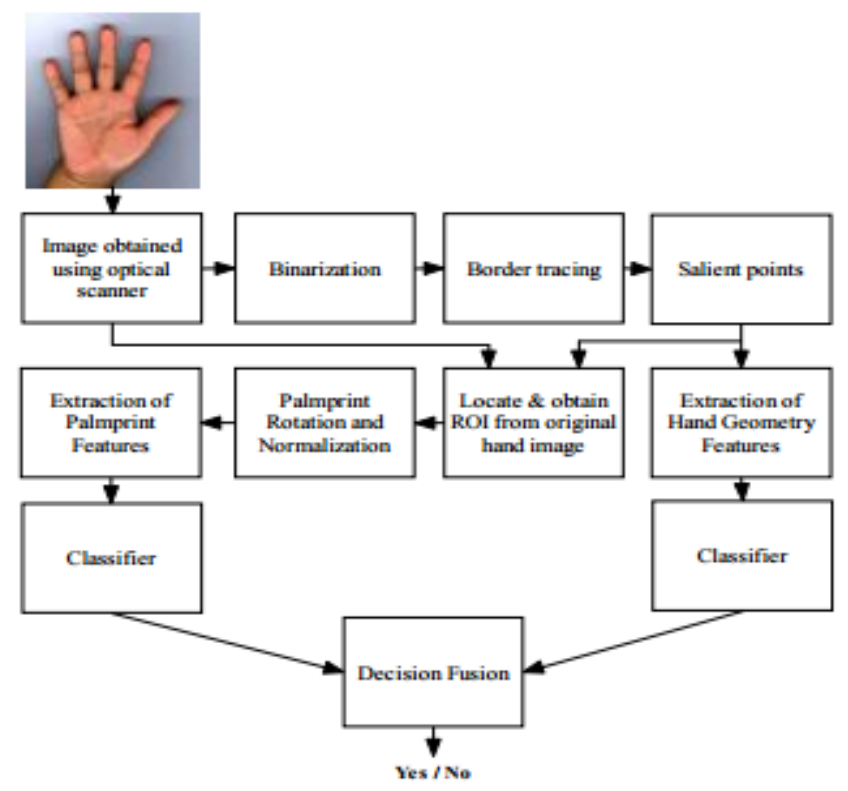

Fig 5: Verification System framework

\section{Image Extraction}

During the image acquiring process, the users are required to stretch their fingers and put their palm straight on the platform of the scanner. The hand images acquired is in 256 RGB colors (8 bits per channel) format. The three color components are important in the pre-processing stage as it can distinguish the background, finger nails, rings and shadow from the hand image. This clear distinction helps to trace the hand image more accurately and reliably.

\subsection{Extraction of salient points}

The hand image acquired from the optical scanner is binarized by using thresholding method [12] to filter the background and shadow from the image. The border tracing algorithm [11] is used to obtain all the vertical coordinates of the border pixels that represents the signature of the hand contour, $f(i)$ where $i$ is the array index. The hand contour signature is then blocked into nonoverlapping frames of 10 samples to check for existence of stationary points in each frame, where their absolute values exceed a predefined threshold, $T s=25$. Hence, the nine salient points with five valleys and four peaks (see Figure. 6) which represent the tips and roots of the fingers are detected respectively. These nine salient points serves as the reference points to measure the length, width and height of the fingers and palm, and also used to detect ROI.

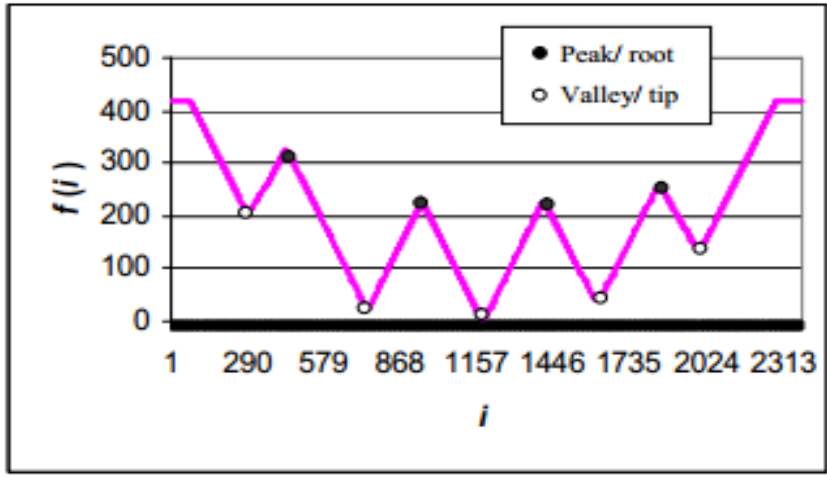

Fig 6. Hand contour signature plot against index: 


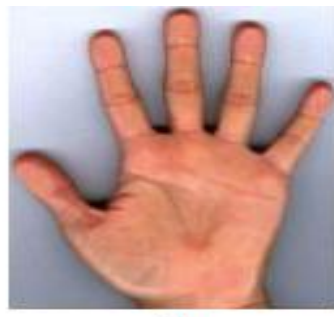

(a)

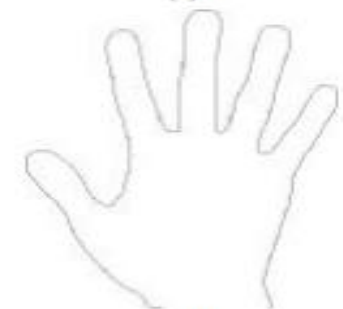

(c)

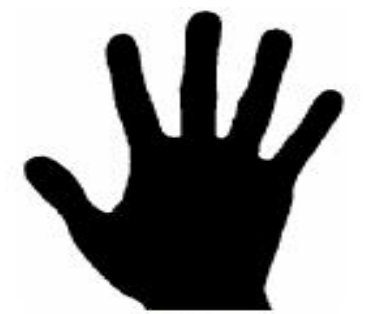

(b)

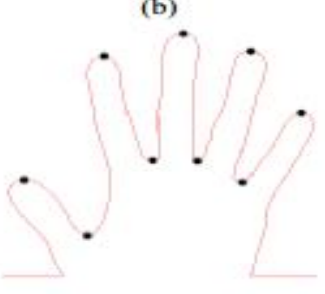

(d)

Fig 7: Salient point detection process, (a) Original hand image acquired from scanner, (b) binarized, image, (c) hand contour,

(d) nine salient points that represent the tips and roots of the fingers.

\subsection{Extraction of ROI}

For palmprint verification system, the ROI is located based on the salient points by using right-angle coordination system [13]. After obtaining the outline of the ROI, the image is cropped and rotated (see Figure 8 ). As the size of ROI vary from hand to hand (depending on the width of the hand), there is a need to resize all of them to a fixed size. In this research, the ROIs are resized to $200 \times 200$ pixels.

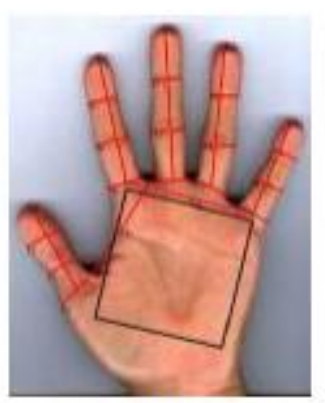

(a)

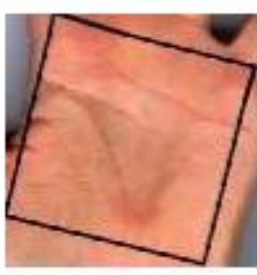

(b)

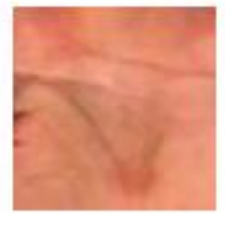

(c)

Fig 8 : ROI extraction process, (a) ROI detection based on salient points, (b) ROI crop, (c) rotation and normalization.

IV.

\section{Feature Extraction}

\subsection{Extraction of hand geometry features}

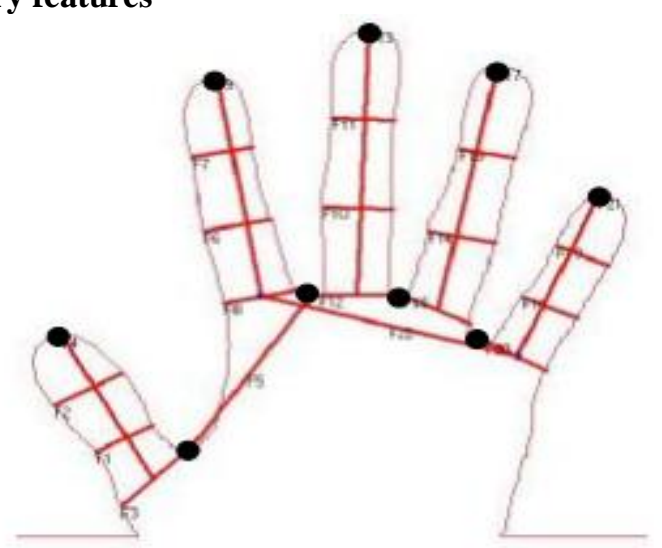

Fig 9 : Hand geometry features. 
Based on the salient points obtained, a automated hand geometry measurement technique proposed in [14] is used to extract the finger lengths, widths and the relative location of the crucial features like knuckles and other joints (as shown in Figure 9). These hand features are important in order to construct a unique pattern for each person. The measuring process generates a feature vector that is an array consists of $\mathrm{N}$ feature values as shown in equation 1 .

$$
\mathrm{Ng}=+57(1)
$$

Where $\mathrm{g}$ is the number of segments that is set for each finger and 7 is the number of features that are obtained from the height of the fingers and width of the palm. For illustration, Figure 5 depicts the case where $g=3$ yield $\mathrm{N}=22$ features

\subsection{Extraction of palmprint features}

For palmprint extraction module in this system, Linear Discriminant Analysis, also known as Fisher Discriminant Analysis (FDA), [15] is used to extract the important palmprint feature from the hand images. FDA maximizes the ratio of between-class scatter to that of within-class scatter. In other words, it projects images such that images of the same class are close to each other while images of different classes are far apart. The basis vectors calculated by Fisher Discriminant create the Fisher Discriminant subspace, which is also called Fisherpalms in this paper.

More formally, consider a set of $\mathrm{M}$ palmprint images having $\mathrm{c}$ classes of images, with each class containing $n$ set images, $\mathrm{i}, \mathrm{i} 1, \mathrm{i} 2, \ldots$ in . Let the mean of images in each class and the total mean of all images be represented by $\mathrm{mc}$ and $\mathrm{m}$, respectively, the images in each class are centered as

$$
\phi_{n}^{c}=i_{n}^{c}-\widetilde{m}_{c} \text { And the class mean is centered as } \omega_{c}=\widetilde{m}_{c}-m
$$

The centered images are then combined side by side into a data matrix. By using this data matrix, an orthonormal basis $U$ is obtained by calculating the full set of eigenvectors of the covariance matrix $\phi_{n}^{c T} \phi_{n}^{c}$. The centered images are then projected into this orthonormal basis as follow

$$
\widehat{\phi_{n}^{c}}=U^{T} \phi_{n}^{c}
$$

The centered means are also projected into the orthonormal basis as-

$$
\widehat{\omega}_{c}=U^{T} \omega_{c}
$$

Based on this information, the within class scatter matrix $S_{W}$ is calculated as-

$$
S_{W}=\sum_{j=1}^{c} \sum_{k=1}^{n_{j}} \widehat{\phi}_{\phi_{k}{ }_{\phi}^{j}}{ }_{k}^{T}
$$

and the between class scatter matrix $S_{B}$ is calculated as

$$
S_{B}=\sum_{j=1}^{C} n_{j} \tilde{\omega}_{j} \tilde{\omega}_{j}^{T}
$$

The generalized eigen vectors $\mathrm{V}$ and eigenvalues $\lambda$ of the within class and between class scatter matrix are solved as follow:

$$
S_{B} V=\lambda S_{W} V
$$

The eigenvectors are sorted according to their associated eigenvalues. The first ${ }^{M-1}$ eigenvectors are kept as the Fisher basis vectors. The rotated images $\alpha_{M}$ where $\alpha_{M}=U^{T} i_{M}$ are projected into the orthonormal basis by

$$
\varpi_{n j}=U^{T} \alpha_{j}
$$

where $n=1, \ldots, M$ and $j=1, \ldots, M-1$. 


\section{Fusion Strategies}

The decision level fusion is selected over feature fusion because matching scores has the lowest data complexity and fusion at decision level often achieves better overall authentication performance $[18,19]$. In the proposed system, we adopt SVM as it is a type of machine learning technique that learns the decision surface to separate the two classes of genuine and imposters through a process of discrimination. It also has good generalization characteristics and has been proven to be a successful classifier on several classical pattern recognition problems [16,17]. Two other combined classifiers, namely sum rule and weighted sum rule are used to compare with the proposed fusion method.

\subsection{Sum Rule}

The summation of both single-modals classifiers matching score or distance is calculated as

$$
S=P_{m s}+H_{m s}
$$

$P_{m s}$ and $H_{m s}$ represent the matching score of palm-print and hand geometry respectively and output the class with the smallest value of $S$.

\subsection{Weighted Sum Rule}

There exists different classifiers with different performance, thus weights can be formed to combine the individual classifiers. Since there is only two single-modal biometrics used in our system, the weighted sum

$$
S_{w}=w P_{m s}+(1-w) H_{m s}
$$

\subsection{Support Vector Machine}

The classification problem in the proposed system can be restricted to two class problem which are genuine and imposter without loss of generality. The goal of using SVM is to separate those two classes by hyper planes, which gives the maximum margin [20]. The support vectors are determined through numerical optimization during the training phase. The Lagrangian (Wolfe) dual objective function for maximal margin separation is given as

$$
L_{D}=\min _{\alpha} \frac{1}{2} \sum_{i=1}^{N} \sum_{j=1}^{N} \alpha_{i} \alpha_{j} d_{i} d_{j} K\left(x_{i}, x_{j}\right)-\sum_{i=1}^{N} \alpha_{i}
$$

where $\mathrm{N}$ is the number of training samples $\alpha_{i}$ and $\alpha_{j}$ are constants determined from training $d_{i}$ and ${ }^{d} j$ is the class indicator (for example, class 1 for genuine and class 2 for imposters) associated with each support vectors, $K\left(x_{i}, x_{j}\right)$ is kernel function performing the non-linear mapping into feature space ${ }^{\mathrm{x}_{i}}$ and ${ }^{\mathrm{x}_{j}}$ are support vectors obtained from the matching scores of the two individual classifiers. Equation (12) is subject to fulfill the following condition,

$$
\begin{gathered}
0 \leq \alpha_{i} \leq C \text { for } i=1,2,3 \ldots, N \text { and } \\
\sum_{i=1}^{N} \alpha_{i} d_{i}=0
\end{gathered}
$$

The kernel function plays an essential role to enable operations to be performed in the input space rather than the high dimensional feature space to achieve better separability between two classes. Two types of kernel functions, the polynomial and Gaussian RBF are being experimented. The polynomial kernel function is formally describe as

$$
K\left(x_{i}, x_{j}\right)=\left(\left(x_{i} \cdot x_{j}\right)+1\right)^{d}
$$

Where $d>0$ represent a constant for the function's degree. On the other hand, RBF kernel function has the Gaussian form of

$$
K\left(x_{i}, x_{j}\right)=\exp \left(-\frac{\left(x_{i}-x_{j}\right)^{2}}{2 \sigma^{2}}\right)
$$

Where ${ }^{\sigma>0}$ is a constant that defines the kernel width. 


\section{Conclusion}

In this paper, a prototype of bimodal biometrics system by using single sensor has been developed. The fusion of two individual biometrics matching scores has significantly reduce the equal error rate of FAR and FRR. The proposed fusion method by using SVM with RBF kernel has been compared with palmprint and hand geometry individual classifiers and two combined classifiers, namely non-weighted sum rule and weighted sum rule.

\section{References}

[1] W.K. Kong and D. Zhang, "Palm print texture analysis based on low-resolution images for personal authentication", in Proceedings of 16th International Conference on Pattern Recognition, vol. 3, pp. 807-810, 2002.

[2] A. Kong, D. Zhang and G. Lu, "A study of identical twins palm print for personal verification", Pattern Recognition, vol. 39, no. 11, pp. 2149-2156, 2006.

[3] [A.W.K. Kong and D. Zhang, "Competitive Coding scheme for palmprint verification", in Proceedings of International Conference on Pattern Recognition, vol. 1, pp. 520-523, 2004.

[4] A. Kong and D. Zhang, "Palm print identification using feature-level fusion", Pattern Recognition, vol. 39, no. 3, pp. 478-487, 2006.

[5] A. Kong, D Zhang and M. Kamel, “A study of brute-force break-ins of a palmprint verification system”, IEEE Transactions on Systems, Man and Cybernetics, Part B, vol. 36, no. 5, pp. 1201-1205, 2006.

[6] Kong, K.H. Cheung, D. Zhang, M. Kamel and J. You, “An analysis of Biohashing and its variants", Pattern Recognition, vol. 39, no. 7, pp. 1359-1368, 2006.

[7] D. Zhang, W.K. Kong, J. You and M. Wong, "On- line palm print identification", IEEE Transactions on Pattern Analysis and Machine Intelligence, vol. 25, no. 9, pp. 1041- 1050, 2003.

[8] T. Connie, A.T.B. Jin, M.G.K. Ong and D.N.C. Ling, “An automated palmprint recognition system”, Image and Vision Computing, vol. 23, no. 5, pp. 501-515, 2005.

[9] C.C. Han, "A hand-based personal authentication using a coarse-to-fine strategy", Image and Vision Computing, vol. 22, no. 11 , pp. 909-918, 2004.

[10] C.C. Han, H.L. Cheng, C.L. Lin and K.C. Fan, "Personal authentication using palm-print features", Pattern Recognition, vol. 36, no. 2, pp. 371-381, 2003.

[11] Y.H. Pang, T. Connie, A. Jin and D. Ling, "Palmprint authentication with Zernike moment invariants", in Proceedings of the 3rd IEEE International Symposium on Signal Processing and Information Technology, pp. 199-202, 2003.

[12] X. Wu, D. Zhang and K. Wang, "Fisherpalms based palmprint recognition", Pattern Recognition Letters, vol. 24, no, 15, pp. 2829-2838, 2003.

[13] G. Lu, D. Zhang and K. Wang, "Palmprint recognition using eigenpalms features", Pattern Recognition Letters, vol. 24, no. 9, pp. $1463-1467,2003$

[14] Lu, X., Wang, Y., and Jain, A.K. "Combining Classifiers for Face Recognition", Proc. ICME 2003, IEEE International Conference on Multimedia \& Expo, vol. III, pp. 13-16, Baltimore, MD, July 6-9, 2003.

[15] Poh, N., Samy, B., Jerzy, K. "IDIAP Research Report: A Multi-sample Multi-source Model for Biometric Authentication", April 2002 .

[16] Issam, E.-N., Yang, Y., Miles N.W., Nikolas, P.G., and Robert, N. "Support Vector Machine Learning for Detection of Microcalcifications in Mammograms", IEEE International Symposium on Biomedical Imaging, Washington D.C., July 2002.

[17] Andrew, T.B.J., Samad, S.A., and Hussain, A. 2002. "Fusion Decision for a Bimodal Biometric Verification System Using Support Vector Machine and Its Variations.” ASEAN Journal on Science and Technology for development, 19(1):1-16.

[18] Steve, G. "ISIS Technical Report: Support Vector Machine for Classification and Regression", Image Speeh \& Intelligent System Group University of Southhampton.

[19] Tee, C., Michael, G.K.O., Andrew, T.B.J., and David, N.C.L. "An Automated Biometric Palmprint Verification System", ISCIT 2003, pp. 714-719, Songkla, Thailand.

[20] Peter, N.B., Hespanha, J.P., and David, J.K. "Eigenfaces vs. Fisherfaces: Recognition Using Class Specific Linear Projection", IEEE Transactions on Pattern Analysis and Machine Intelligence, vol. 19, no. 7, July 1997. 